\title{
Scalar-graviton amplitudes
}

\section{N.E.J. Bjerrum-Bohr, ${ }^{a}$ Andrea Cristofoli, ${ }^{a}$ Poul H. Damgaard ${ }^{a}$ and Humberto Gomez ${ }^{a, b}$}

\author{
${ }^{a}$ Niels Bohr International Academy and Discovery Center, \\ The Niels Bohr Institute, University of Copenhagen, \\ Blegdamsvej 17, DK-2100 Copenhagen, Denmark \\ ${ }^{b}$ Facultad de Ciencias, Basicas Universidad, \\ Santiago de Cali, Calle $5 N^{\circ}$ 62-00 Barrio Pampalinda, Cali, Valle, Colombia \\ E-mail: bjbohr@nbi.dk, a.cristofoli@nbi.ku.dk, phdamg@nbi.dk, \\ humberto.gomez@nbi.ku.dk
}

ABSTRACT: Using the CHY-formalism and its extension to a double cover we provide covariant expressions for tree-level amplitudes with two massive scalar legs and an arbitrary number of gravitons in $D$ dimensions. Using unitarity methods, such amplitudes are needed inputs for the computation of post-Newtonian and post-Minkowskian expansions in classical general relativity.

Keywords: Scattering Amplitudes, Duality in Gauge Field Theories, Field Theories in Higher Dimensions

ArXiv ePrint: 1908.09755 


\section{Contents}

1 Introduction 1

2 Prelude: two massive scalars and $n$ gluons 2

2.1 Massive Yang-Mills amplitudes 2

$\begin{array}{lll}2.2 & \text { Turning massive gluons into scalars } & 6\end{array}$

$\begin{array}{llr}3 & \text { Kleiss-Kuijf decomposition } & 9\end{array}$

4 Two massive scalars and gravitons $\quad 12$

5 Conclusion $\quad 13$

$\begin{array}{ll}\text { A Higher-point Yang-Mills amplitudes with massive legs } & 14\end{array}$

$\begin{array}{ll}\text { B Longitudinal contributions } & 16\end{array}$

\section{Introduction}

Recently it has been realized that modern methods for amplitude computations at loop level may provide a powerful new way to compute post-Newtonian and post-Minkowskian expansions in classical general relativity [1-21]. This builds on the observation that the quantum mechanical scattering matrix for matter interacting gravitationally contains classical pieces at arbitrarily high order in the loop expansion [22-24] and the fact that the sought-for longdistance contributions are non-analytic in the exchanged momentum [25-27], thus making them straightforwardly accessible through unitarity cuts. All needed contributions being classical, one would not expect it to be necessary to regularize the loops dimensionally. However, since infrared 'super-classical' (see, e.g., ref. [24]) terms appear at intermediate steps it is nevertheless convenient to use dimensional regularization.

For the scattering of two massive objects at large distances the needed tree-level amplitudes are those of two massive scalars and, at $n$-loop order, $(n+1)$ on-shell gravitons. Using the Kawai-Lewellen-Tye (KLT) relations [28-32] these can conveniently be constructed from the corresponding amplitudes with the $(n+1)$ gravitons replaced by gluons, amplitudes that are given in the literature on the basis of recursion relations [33, 34] in four space-time dimensions, using the spinor-helicity formalism. More recently, Naculich [35, 36] has suggested an alternative and more direct method for the computation of such amplitudes based on the Cachazo-He-Yuan (CHY) formalism [37, 38] (see also ref. [60]). One advantage of using the CHY-formalism is that it immediately provides the amplitudes 'covariantly', in terms of general polarization tensors for the gravitons, and hence not restricted to four space-time dimensions. 
From a practical point of view, it suffices to evaluate amplitudes with two scalar legs and $(n+1)$ gluons and subsequently turning them into scalar-graviton amplitudes by KLT-squaring. This is our approach here. One key point of the present calculation is the computation of a factorized expression for the amplitudes of two massive scalars coupled to Yang-Mills theory, expressing them as sums over lower-point amplitudes which are combinations of scalar-gluon amplitudes and pure gluon amplitudes. Each of these has one gluon leg off-shell and an associated polarization vector of both transverse and longitudinal components. In this way, we can iteratively construct amplitudes of an arbitrarily high order. Crucial for this factorized form is the insight gained from the double-cover version [39-43] of the CHY-formalism. This double-cover description naturally splits amplitudes into two lower-point amplitudes, each with one leg off-shell. These vector currents, contracted with polarization vectors, are glued together by the polarization sum. A subtlety here is the contribution from longitudinal modes that need to be dealt with carefully. Useful relations that short-cut the evaluations of some of the color-ordered amplitudes needed for the recursive evaluation of higher $n$-point amplitudes are provided by simple identities [44-46] among these partly massive amplitudes.

The outline of this paper is as follows. In sections 2 and 3 we show how to compute amplitudes with two scalars and $n$ gluons using different methods. In section 4 we briefly discuss the straightforward application of Kawai-Lewellen-Tye relations to replace the gluons with gravitons. Some technical details and a proof of an important theorem regarding vanishing longitudinal contributions are provided in appendices.

\section{Prelude: two massive scalars and $n$ gluons}

We first present a simple way to obtain explicit expressions for the scattering amplitudes of two massive scalars and $n$ gluons. Since our method is based on the CHY approach, we give a very brief review of this formalism. We then apply the factorization method developed in [43] to obtain, up to six-point, analytical expressions for the scattering of gluons where two of them, suitably defined, are massive. Next, we turn the two massive gluons into massive scalars, thus providing the scattering amplitudes for two massive scalars and in principle any number of massless gluons.

\subsection{Massive Yang-Mills amplitudes}

We start by presenting a simple recursive formula that computes pure Yang-Mills amplitudes with up to three massive gluons. The method we will use was developed by one of us in a different context [39, 43]. We shall show explicit expressions up to six points but it is straightforward to extend the method to any higher number of external legs. In the following, we will denote massive particles with the capital letter " $P_{\alpha}$ " and the massless ones with the lower-case letter " $k_{a}$ ". Unless otherwise mentioned we will work under the assumption of implicit momentum conservation,

$$
K_{1}+K_{2}+\cdots+K_{n}=0
$$


Let us first recall how to extend the CHY approach to the massive case following the method of Naculich $[35,36]$. We have $\left\{P_{1}, \ldots, P_{i}\right\}$ as momenta of the massive particles $\left(P_{\alpha}^{2} \neq 0\right)$ and $\left\{k_{i+1}, \ldots, k_{n}\right\}$ as momenta the massless gluons $\left(k_{a}^{2}=0\right)$. A generic momentum vector is thus $K_{A} \in\left\{P_{1}, \ldots, P_{i}, k_{i+1}, \ldots, k_{n}\right\}$. We define as well

$$
\begin{aligned}
P_{A B \ldots D} & \equiv K_{A}+K_{B}+\cdots+K_{D}, \\
P_{A: A+j} & \equiv K_{A}+K_{A+1}+\cdots+K_{A+j} .
\end{aligned}
$$

The modified CHY scattering equations are then given by

$$
S_{A}=\sum_{\substack{B=1 \\ B \neq A}}^{n} \frac{2 K_{A} \cdot K_{B}+2 \Delta_{A B}}{\sigma_{A B}}=0, \quad A=1,2, \ldots, n,
$$

where the matrix $\Delta_{A B}$ is still to be determined. In order to guarantee $\operatorname{SL}(2, \mathbb{C})$ invariance, i.e., $\sum_{A=1}^{n} \sigma_{A}^{m} S_{A}=0$ for $m=0,1,2$, the matrix $\Delta_{A B}$ must be symmetric, $\Delta_{A B}=\Delta_{B A}$, and it must satisfy the conditions

$$
\begin{array}{ll}
\sum_{\substack{\beta=1 \\
\beta \neq a}}^{i} \Delta_{\alpha \beta}+\sum_{b=i+1}^{n} \Delta_{\alpha b}=P_{\alpha}^{2}, & \alpha=1, \ldots, i, \\
\sum_{\beta=1}^{i} \Delta_{a \beta}+\sum_{\substack{b=i+1 \\
b \neq a}}^{n} \Delta_{a b}=0, & a=i+1, \ldots, n .
\end{array}
$$

Since we are interested in at most up to three massive gluons of momenta $\left\{P_{1}, P_{2}, P_{3}\right\}$, it is sufficient to consider only $\Delta_{12}, \Delta_{13}, \Delta_{23}$. Therefore, we have the simple conditions

$$
\begin{aligned}
& \Delta_{12}+\Delta_{13}=P_{1}^{2}, \\
& \Delta_{12}+\Delta_{23}=P_{2}^{2}, \\
& \Delta_{13}+\Delta_{23}=P_{3}^{2},
\end{aligned}
$$

that have a unique solution given by

$$
\Delta_{12}=\frac{P_{1}^{2}+P_{2}^{2}-P_{3}^{4}}{2}, \quad \Delta_{13}=\frac{P_{1}^{2}-P_{2}^{2}+P_{3}^{4}}{2}, \quad \Delta_{23}=\frac{-P_{1}^{2}+P_{2}^{2}+P_{3}^{4}}{2} .
$$

When two masses are degenerate, e.g., $P_{1}^{2}=P_{2}^{2} \neq 0$ and $P_{3}^{2}=0$, it is straightforward to see from (2.6) that $\Delta_{12}=P_{1}^{2}$ and $\Delta_{13}=\Delta_{23}=0$, which, not surprisingly, is in agreement with the one-loop scattering equations formulated in refs. [40, 47-49]. On the other hand, when only one of the legs is massive, e.g. $P_{1}^{2} \neq 0$ and $P_{2}^{2}=P_{3}^{2}=0$, then $\Delta_{12}=P_{1}^{2} / 2$, $\Delta_{13}=P_{1}^{2} / 2$ and $\Delta_{23}=-P_{1}^{2} / 2$, i.e., in order to describe one massive particle it is necessary to use at least three $\Delta_{A B}$ parameters.

After having described the massive scattering equations let us now remind that the CHY prescription for color ordered amplitudes of the scattering of gluons at tree-level is given by [35-37, 50]

$$
A_{n}\left(P_{1}, \ldots, P_{i}, i+1, \ldots, n\right)=\int d \mu_{n} \mathrm{PT}(1,2, \ldots, n) \times \operatorname{Pf}^{\prime} \Psi_{n},
$$


where $d \mu_{n}$ is the usual CHY measure

$$
d \mu_{n}=\left(\sigma_{j k} \sigma_{k l} \sigma_{l j}\right) \prod_{\substack{A=1 \\ A \neq j, k, l}}^{n} d \sigma_{A} \times\left(\sigma_{m r} \sigma_{r s} \sigma_{s m}\right) \prod_{\substack{B=1 \\ B \neq m, r, s}}^{n} \delta\left(S_{B}\right)
$$

and $\mathrm{PT}(1, \ldots, n)$ and $\mathrm{Pf}^{\prime} \Psi_{n}$ are the usual Parke-Taylor and reduced Pfaffian factors

$$
\mathrm{PT}(1, \ldots, n) \equiv \frac{1}{\sigma_{12} \sigma_{23} \cdots \sigma_{n 1}}, \quad \operatorname{Pf}^{\prime} \Psi_{n} \equiv \frac{(-1)^{A+B}}{\sigma_{A B}} \operatorname{Pf}\left[\left(\Psi_{n}\right)_{A B}^{A B}\right]
$$

The $2 n \times 2 n$ matrix, $\Psi_{n}$, is defined as

$$
\Psi_{n} \equiv\left(\begin{array}{cc}
\mathrm{A} & -\mathrm{C}^{\mathrm{T}} \\
\mathrm{C} & \mathrm{B}
\end{array}\right)
$$

with,

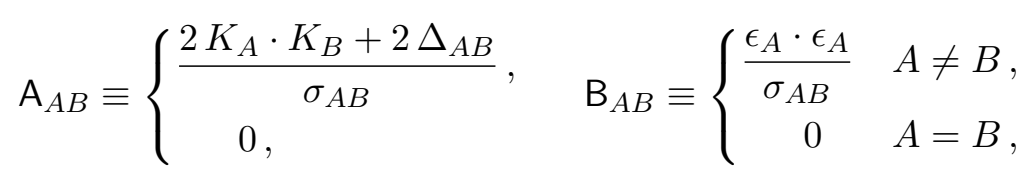

and

$$
\mathrm{C}_{A B} \equiv \begin{cases}\frac{\sqrt{2} \epsilon_{A} \cdot K_{B}}{\sigma_{A B}}, & A \neq B \\ -\sum_{\substack{C=1 \\ n \\ C \neq A}}^{n} \frac{\sqrt{2} \epsilon_{A} \cdot K_{C}}{\sigma_{A C}}, & A=B\end{cases}
$$

The matrix, $\left(\Psi_{n}\right)_{A B}^{A B}$, denotes the reduced matrix obtained by removing the rows and columns $A, B$ from $\Psi_{n}$, where $1 \leq A<B \leq n$.

Since we are interested in the case of at most three massive particles of momenta $\left\{P_{1}, P_{2}, P_{3}\right\}$ we can avoid dealing with the $\Delta_{A B}$-matrix in the scattering equations altogether by choosing the labels $\{j, k, l\}$ and $\{m, r, s\}$ in (2.8) to match with the massive ones, i.e., $\{j, k, l\}=\{m, r, s\}=\{1,2,3\}$.

It is useful to recall that the reduced Pfaffian $\left(\operatorname{Pf}^{\prime} \Psi_{n}=\frac{(-1)^{A+B}}{\sigma_{A B}} \operatorname{Pf}\left[\left(\Psi_{n}\right)_{A B}^{A B}\right]\right)$ is independent of the choice of $A$ and $B$, and that the $\operatorname{SL}(2, \mathbb{C})$ symmetry is guaranteed by the transversality of the external polarization vectors, $\left(\epsilon_{C} \cdot K_{C}\right)=0$. However, we note that the terms $C_{A A}$ and $C_{B B}$ do not appears in the reduced matrix, $\left(\Psi_{n}\right)_{A B}^{A B}$. It follows that the transversality conditions on $\epsilon_{A}$ and $\epsilon_{B}$ are not needed to obtain an integrand invariant under the action of $\operatorname{SL}(2, \mathbb{C})[51]$. We can therefore consistently define the integral with these two legs being off mass-shell and with arbitrary polarization vectors for $\left(\epsilon_{A} \cdot K_{A}\right) \neq 0$ and $\left(\epsilon_{B} \cdot K_{B}\right) \neq 0$. We now use the double-cover method ref. [43] to obtain compact recursive expressions for these massive and/or off-shell scattering amplitudes as defined above. The results clearly reduce to the usual expressions when all external legs are massless and on-shell.

First, let us consider the basic building block of three legs. We take all three particles to be massive and choose the polarization vectors $\epsilon_{1}$ and $\epsilon_{2}$ as not necessarily transverse 
so that we do not impose $\left(\epsilon_{1} \cdot P_{1}\right)=0=\left(\epsilon_{2} \cdot P_{2}\right)$. We are going to denote with a bold source in the amplitude (as in [35-37, 43]), e.g.

$$
A_{n}\left(\ldots, \boldsymbol{P}_{\alpha}, \ldots, \boldsymbol{P}_{\beta}, \ldots\right),
$$

the rows/columns that are removed from its reduced Pfaffian. In the above amplitude the reduced Pfaffian is given by, $\operatorname{Pf}^{\prime} \Psi_{n}=\frac{(-1)^{\alpha+\beta}}{\sigma_{\alpha \beta}} \operatorname{Pf}\left[\left(\Psi_{n}\right)_{\alpha \beta}^{\alpha \beta}\right]$. Particles $P_{\alpha}$ and $P_{\beta}$ can thus be off-shell, so that $\left(\epsilon_{\alpha} \cdot P_{\alpha}\right) \neq 0$ and $\left(\epsilon_{\beta} \cdot P_{\beta}\right) \neq 0$.

Therefore, using the CHY prescription given in (2.7) one has

$$
\begin{aligned}
A_{3}\left(\boldsymbol{P}_{1}, \boldsymbol{P}_{2}, P_{3}\right) & =\left(\sigma_{12} \sigma_{23} \sigma_{23}\right)^{2} \mathrm{PT}(1,2,3) \frac{(-1)}{\sigma_{12}} \operatorname{Pf}\left[\begin{array}{cccc}
0 & -\frac{\epsilon_{1} \cdot \sqrt{2} P_{3}}{\sigma_{13}} & -\frac{\epsilon_{2} \cdot \sqrt{2} P_{3}}{\sigma_{23}} & -\mathrm{C}_{33} \\
\frac{\epsilon_{1} \cdot \sqrt{2} P_{3}}{\sigma_{13}} & 0 & \frac{\epsilon_{1} \cdot \epsilon_{2}}{\sigma_{12}} & \frac{\epsilon_{1} \cdot \epsilon_{3}}{\sigma_{13}} \\
\frac{\epsilon_{2} \cdot \sqrt{2} P_{3}}{\sigma_{23}} & \frac{\epsilon_{2} \cdot \epsilon_{1}}{\sigma_{21}} & 0 & \frac{\epsilon_{2} \cdot \epsilon_{3}}{\sigma_{23}} \\
\mathrm{C}_{33} & \frac{\epsilon_{3} \cdot \epsilon_{1}}{\sigma_{31}} & \frac{\epsilon_{3} \cdot \epsilon_{2}}{\sigma_{32}} & 0
\end{array}\right] \\
& =\sqrt{2}\left\{\left(\epsilon_{1} \cdot \epsilon_{2}\right)\left(\epsilon_{3} \cdot P_{1}\right)-\left(\epsilon_{2} \cdot \epsilon_{3}\right)\left(\epsilon_{1} \cdot P_{3}\right)+\left(\epsilon_{3} \cdot \epsilon_{1}\right)\left(\epsilon_{2} \cdot P_{3}\right)\right\},
\end{aligned}
$$

where we have used

$$
\mathrm{C}_{33}=-\sqrt{2}\left(\frac{\epsilon_{3} \cdot P_{1}}{\sigma_{31}}+\frac{\epsilon_{3} \cdot P_{2}}{\sigma_{32}}\right)=\sqrt{2}\left(\epsilon_{3} \cdot P_{1}\right) \times \frac{\sigma_{12}}{\sigma_{31} \sigma_{23}},
$$

due to the momentum conservation constraint $P_{1}+P_{2}+P_{3}=0$ and the transversality condition $\left(\epsilon_{3} \cdot P_{3}\right)=0$. Although the amplitude itself is independent of the choice of rows/columns that are removed in the Pfaffian, the intermediate expressions do depend on the choice and we have therefore introduced a notation where we indicate which rows and columns are removed.

We consider next a computation with three massive gluons of momenta $\left\{P_{1}, P_{2}, P_{3}\right\}$ and one massless gluon of momentum $\left\{k_{4}\right\}$. Using the factorization method described in $[41,43]$, this four-point calculation can be expressed in terms of the $A_{3}\left(\boldsymbol{P}_{a}, \boldsymbol{P}_{b}, P_{c}\right)$ building-blocks,

$$
\begin{aligned}
& A_{4}\left(\boldsymbol{P}_{1}, P_{2}, \boldsymbol{P}_{3}, 4\right) \\
& =\sum_{M}\left[\frac{A_{3}\left(\boldsymbol{P}_{34}^{\epsilon^{M}}, \boldsymbol{P}_{1}, P_{2}\right) A_{3}\left(\boldsymbol{P}_{12}^{\epsilon^{M}}, \boldsymbol{P}_{3}, 4\right)}{s_{P_{3}}}+\frac{A_{3}\left(\boldsymbol{P}_{1}, \boldsymbol{P}_{23}^{\epsilon^{M}}, 4\right) A_{3}\left(\boldsymbol{P}_{3}, \boldsymbol{P}_{41}^{\epsilon^{M}}, P_{2}\right)}{s_{4 P_{1}}}\right] \\
& \quad-2 \sum_{L}\left[\frac{A_{3}\left(\boldsymbol{P}_{13}^{\epsilon^{L}}, \boldsymbol{P}_{2}, 4\right)}{s_{P_{2}}} \times A_{3}\left(\boldsymbol{P}_{24}^{\epsilon^{L}}, \boldsymbol{P}_{1}, P_{3}\right)\right]
\end{aligned}
$$

where the notation $P_{i}^{\epsilon^{M}}\left(P_{i}^{\epsilon^{L}}\right)$ means the particle with momentum $P_{i}$ has as polarization vector $\epsilon_{i}^{M}\left(\epsilon_{i}^{L}\right)$. The sums over the polarizations are given by the relations

$$
\begin{aligned}
\sum_{M} \epsilon_{i}^{M \mu} \epsilon_{j}^{M \nu} & =\eta^{\mu \nu}, \\
\sum_{L} \epsilon_{i}^{L \mu} \epsilon_{j}^{L \nu} & =\frac{P_{i}^{\mu} P_{j}^{\nu}}{P_{i} \cdot P_{j}+P_{1}^{2}-P_{3}^{2}} .
\end{aligned}
$$


The unusual normalization factor of the longitudinal modes is precisely what is needed to recover the correct four-point amplitude $[42,52]$. The polarization vectors of all massive on-shell legs of course still satisfy $\epsilon_{i} \cdot P_{i}=0$. Using that condition it is easy to see that the last term in (2.16) evaluates to

$$
-2 \sum_{L}\left[\frac{A_{3}\left(\boldsymbol{P}_{13}^{\epsilon^{L}}, \boldsymbol{P}_{2}, 4\right)}{s_{P_{2}}} \times A_{3}\left(\boldsymbol{P}_{24}^{\epsilon^{L}}, \boldsymbol{P}_{1}, P_{3}\right)\right]=\left(\epsilon_{1} \cdot \epsilon_{3}\right)\left(\epsilon_{2} \cdot \epsilon_{4}\right) .
$$

The full four-point amplitude is thus remarkably simple.

Finally, in order to calculate higher-point amplitudes we will also need $A_{4}\left(\boldsymbol{P}_{1}, \boldsymbol{P}_{2}, P_{3}, 4\right)$. Using the BCJ-like identity [44-46],

$$
s_{4 P_{3}} \mathrm{PT}(3,4,1,2)+s_{4 P_{13}} \mathrm{PT}(3,1,4,2)=0,
$$

it is straightforward to deduce

$$
A_{4}\left(\boldsymbol{P}_{1}, \boldsymbol{P}_{2}, P_{3}, 4\right)=-\left(1+\frac{s_{4 P_{1}}}{s_{4 P_{3}}}\right) \times A_{4}\left(\boldsymbol{P}_{1}, P_{3}, \boldsymbol{P}_{2}, 4\right) .
$$

The calculation of higher-point amplitudes with massive gluons now proceeds recursively. We illustrate a few cases in the appendix.

\subsection{Turning massive gluons into scalars}

Now, using the prescriptions of Naculich [35, 36] and Cachazo, He, and Yuan [38] we can compute the amplitudes of interest which also involve massive scalar legs. The basic idea is to consider the massive gluon theory in one extra dimension (i.e., in $D+1$ dimensions) with "polarizations" and momenta of massive scalars chosen to be

$$
\left.\begin{array}{ll}
P_{1}^{\mu}=\left(\overrightarrow{p_{1}}, 0\right), & \epsilon_{1}^{\mu}=(\overrightarrow{0}, 1) \\
P_{n}^{\mu}=\left(\overrightarrow{p_{n}}, 0\right), & \epsilon_{n}^{\mu}=(\overrightarrow{0}, 1)
\end{array}\right\} \quad \text { Massive scalars }\left(P_{1}^{2}=P_{n}^{2}=m^{2}\right),
$$

In this set-up all external particles satisfy $P_{i} \cdot \epsilon_{i}=0=k_{a} \cdot \epsilon_{a}$ and, additionally, it is easy to see that $\Delta_{1 n}=\Delta_{n 1}=m^{2}$ in Naculich's notation as a consequence of equation (2.6). The CHY prescription for the scattering of two massive scalars with $n-2$ gluons can thus be written as

$$
A_{n}\left(1_{\varphi}, 2_{g}, \ldots,(n-1)_{g}, n_{\varphi}\right)=\int d \mu_{n} \operatorname{PT}(1,2, \ldots, n) \times\left.\operatorname{Pf}^{\prime} \Psi_{n}\right|_{\epsilon_{1}, \epsilon_{n}=(\overrightarrow{0}, 1)},
$$

where the massive scattering equations, the reduced Pfaffian and the measure as defined above. It is useful to note that these ordered amplitudes are invariant under cyclic permutations, i.e.,

$$
A_{n}\left(1,2, \ldots, \boldsymbol{P}_{\alpha}, \ldots, \boldsymbol{P}_{\beta}, \ldots, n\right)=A_{n}\left(n, 1,2, \ldots, \boldsymbol{P}_{\alpha}, \ldots, \boldsymbol{P}_{\beta}, \ldots, n-1\right),
$$

and also satisfy

$$
A_{n}\left(1,2, \ldots, \boldsymbol{P}_{\alpha}, \ldots, \boldsymbol{P}_{\beta}, \ldots, n-1, n\right)=(-1)^{n} A_{n}\left(n, n-1, \ldots, \boldsymbol{P}_{\beta}, \ldots, \boldsymbol{P}_{\alpha}, \ldots, 2,1\right) .
$$


As a first step we note that when $P_{1}$ and $P_{2}$ are associated with scalar legs the three-point amplitude reads

$$
\left.A_{3}\left(\boldsymbol{P}_{1}, \boldsymbol{P}_{2}, P_{3}\right)\right|_{\epsilon_{1}, \epsilon_{2}=(\overrightarrow{0}, 1)}=\sqrt{2}\left(\epsilon_{3} \cdot P_{1}\right) .
$$

We illustrate our method by evaluating the four-point function of two massive scalars and two gluons. Using the above conditions and the cyclicity property (2.23) we immediately infer this amplitude from eqs. (2.16):

$$
\begin{aligned}
A_{4}\left(1_{\varphi}, 2_{g}, 3_{g}, 4_{\varphi}\right) & =\left.A_{4}\left(\boldsymbol{P}_{4}, P_{1}, \mathbf{2}, 3\right)\right|_{\epsilon_{1}, \epsilon_{4}=(\overrightarrow{0}, 1)} \\
& =\sum_{M}\left[\frac{A_{3}\left(\boldsymbol{P}_{23}^{\epsilon^{M}}, \boldsymbol{P}_{4}^{\varphi}, P_{1}^{\varphi}\right) A_{3}\left(\boldsymbol{P}_{41}^{\epsilon^{M}}, \mathbf{2}, 3\right)}{s_{23}}+\frac{A_{3}\left(\boldsymbol{P}_{4}^{\varphi}, \boldsymbol{P}_{12}^{\epsilon^{M}}, 3\right) A_{3}\left(\mathbf{2}, \boldsymbol{P}_{34}^{\epsilon^{M}}, P_{1}^{\varphi}\right)}{s_{3 P_{4}}}\right],
\end{aligned}
$$

where the superscript (or subscript) " $\varphi$ " refers to one of the massive scalars. We note that the term (2.19) does not contribute at all, (we shall return to this point later).

Remark. Since $\epsilon_{1}^{\mu}=\epsilon_{4}^{\mu}=(\overrightarrow{0}, 1)$ the contraction relation for the second term in (2.25),

$$
\sum_{M}\left(\epsilon_{P_{34}}^{M} \cdot \epsilon_{1}\right)\left(\epsilon_{P_{12}}^{M} \cdot V\right)=\left(\epsilon_{1} \cdot V\right),
$$

is non-vanishing only when $V^{\mu}$ has a non-zero projection on $\epsilon_{4}$. Therefore, it is equivalent to choosing $\epsilon_{P_{34}}^{M \mu}=\epsilon_{P_{12}}^{M \mu}=(\overrightarrow{0}, 1)$, i.e., the internal lines corresponding to momenta $P_{12}$ and $P_{34}$ turn out to be propagating scalars as expected due to current conservation. In other words,

$$
\sum_{M} \frac{A_{3}\left(\boldsymbol{P}_{4}^{\varphi}, \boldsymbol{P}_{12}^{\epsilon^{M}}, 3\right) A_{3}\left(\mathbf{2}, \boldsymbol{P}_{34}^{\epsilon^{M}}, P_{1}^{\varphi}\right)}{s_{3 P_{4}}}=\frac{A_{3}\left(\boldsymbol{P}_{4}^{\varphi}, \boldsymbol{P}_{12}^{\varphi}, 3\right) A_{3}\left(\mathbf{2}, \boldsymbol{P}_{34}^{\varphi}, P_{1}^{\varphi}\right)}{s_{3 P_{4}}}
$$

The same phenomenon occurs for higher $n$-point amplitudes. Let us now introduce some convenient notation:

$$
\begin{aligned}
F_{A}^{\mu \nu} & \equiv K_{A}^{\mu} \epsilon_{A}^{\nu}-K_{A}^{\nu} \epsilon_{A}^{\mu}, \\
(V W)_{a b} & \equiv V_{a}^{\mu} \eta_{\mu \nu} W_{b}^{\nu},
\end{aligned}
$$

as well as

$$
\begin{aligned}
(V F \ldots F W)_{a A_{1} \ldots A_{j} b} & \equiv V_{a}^{\mu} \eta_{\mu \gamma} F_{A_{1}}^{\gamma \nu} \eta_{\nu \sigma} F_{A_{2}}^{\sigma \alpha} \cdots F_{A_{j}}^{\rho \delta} \eta_{\delta \beta} W_{b}^{\beta} \\
s_{A_{1} A_{2} \ldots A_{j}} & \equiv\left(K_{A_{1}}+K_{A_{2}}+\cdots+K_{A_{j}}\right)^{2}-\left(K_{A_{1}}^{2}+K_{A_{2}}^{2}+\cdots+K_{A_{j}}^{2}\right)
\end{aligned}
$$

where $V_{a}^{\mu}$ and $W_{b}^{\nu}$ are two generic vectors. From (2.17) and (2.14) it is straightforward to compute

$$
\sum_{M} \frac{A_{3}\left(\boldsymbol{P}_{23}^{\epsilon^{M}}, \boldsymbol{P}_{4}^{\varphi}, P_{1}^{\varphi}\right) A_{3}\left(\boldsymbol{P}_{41}^{\epsilon^{M}}, \mathbf{2}, 3\right)}{s_{23}}=\frac{2(\epsilon P)_{21}(\epsilon k)_{32}-2(\epsilon F P)_{231}}{s_{23}},
$$

as well as

$$
\frac{A_{3}\left(\boldsymbol{P}_{4}^{\varphi}, \boldsymbol{P}_{12}^{\varphi}, 3\right) A_{3}\left(\mathbf{2}, \boldsymbol{P}_{34}^{\varphi}, P_{1}^{\varphi}\right)}{s_{3 P_{4}}}=-\frac{2(\epsilon P)_{21}(\epsilon P)_{34}}{s_{P_{1} 2}}
$$


The four-point covariant amplitude of two massive scalars and two gluons is thus given by the simple expression

$$
A_{4}\left(1_{\varphi}, 2_{g}, 3_{g}, 4_{\varphi}\right)=\frac{2(\epsilon P)_{21}(\epsilon k)_{32}-2(\epsilon F P)_{231}}{s_{23}}-\frac{2(\epsilon P)_{21}(\epsilon P)_{34}}{s_{P_{1} 2}} .
$$

Specializing to four dimensions, this is in agreement with the result found in the literature on the basis of the spinor-helicity formalism [34].

In an analogous way, the five-point amplitude becomes

$$
\begin{aligned}
& A_{5}\left(1_{\varphi}, 2_{g}, 3_{g}, 4_{g}, 5_{\varphi}\right)=\left.A_{5}\left(\boldsymbol{P}_{5}, P_{1}, \mathbf{2}, 3,4\right)\right|_{\epsilon_{1}, \epsilon_{5}=(\overrightarrow{0}, 1)} \\
& =(-1) \times \sum_{M}\left[\frac{A_{3}\left(\boldsymbol{P}_{4: 1}^{\epsilon^{M}}, \mathbf{2}, 3\right) A_{4}\left(\boldsymbol{P}_{5}^{\varphi}, P_{1}^{\varphi}, \boldsymbol{P}_{23}^{\epsilon^{M}}, 4\right)}{s_{23}}+\frac{A_{3}\left(\boldsymbol{P}_{2: 4}^{\epsilon^{M}}, \boldsymbol{P}_{5}^{\varphi}, P_{1}^{\varphi}\right) A_{4}\left(\boldsymbol{P}_{51}^{\epsilon^{M}}, \mathbf{2}, 3,4\right)}{s_{234}}\right] \\
& \quad+(-1) \times \frac{A_{3}\left(\mathbf{2}, \boldsymbol{P}_{3: 5}^{\varphi}, P_{1}^{\varphi}\right) \times A_{4}\left(\boldsymbol{P}_{5}^{\varphi}, \boldsymbol{P}_{12}^{\varphi}, 3,4\right)}{s_{34 P_{5}}},
\end{aligned}
$$

where eq. (A.1) has been used. As in the four-point case, the purely longitudinal contributions vanish on account of the orthogonality conditions for the polarization vectors associated with external scalar legs, $\left(\epsilon_{1} \cdot \epsilon_{3}\right)=\left(\epsilon_{5} \cdot \epsilon_{2}\right)=0$. In appendix B, we prove the vanishing of these longitudinal contributions for any number of external gluons.

Applying the identity (2.17) and using (2.14), (2.16) and (2.20) we finally find an explicit covariant expression for $A_{5}\left(1_{\varphi}, 2_{g}, 3_{g}, 4_{g}, 5_{\varphi}\right)$ :

$$
\begin{aligned}
\left(\frac{1}{\sqrt{2}}\right) & \times A_{5}\left(1_{\varphi}, 2_{g}, 3_{g}, 4_{g}, 5_{\varphi}\right)=(\epsilon P)_{21} \times \frac{(\epsilon \epsilon)_{34} s_{34}-2(\epsilon k)_{34}(\epsilon P)_{45}+2(\epsilon k)_{43}(\epsilon P)_{35}}{s_{P_{1} 2} s_{34}} \\
& +\frac{(\epsilon \epsilon)_{23}(\epsilon k)_{43} s_{23}-(\epsilon \epsilon)_{34}(\epsilon P)_{21} s_{23}-(\epsilon \epsilon)_{23}(\epsilon P)_{41} s_{34}-(\epsilon F \epsilon)_{342} s_{23}}{s_{23} s_{34}}+\frac{(\epsilon \epsilon)_{34}(\epsilon P)_{21} s_{4 P_{5}}}{s_{P_{1} 2} s_{34}} \\
& +2(\epsilon P)_{21}(\epsilon P)_{45} \times \frac{(\epsilon P)_{31}+(\epsilon k)_{32}}{s_{P_{1} 2} s_{4 P_{5}}}+(\epsilon P)_{45} \times \frac{2(\epsilon k)_{21}(\epsilon P)_{32}-2(\epsilon k)_{23}(\epsilon P)_{31}-(\epsilon \epsilon)_{23} s_{23}}{s_{23} s_{4 P_{5}}} \\
& +\frac{s_{P_{1} 2}(\epsilon F \epsilon)_{243}+(\epsilon \epsilon)_{23}(\epsilon k)_{43} s_{P_{1} 2}-(\epsilon \epsilon)_{34}(\epsilon P)_{21} s_{P_{1} 4}-(\epsilon \epsilon)_{34}(\epsilon P)_{25} s_{P_{1} 4}+(\epsilon \epsilon)_{34}(\epsilon P)_{21} s_{23}}{s_{34} s_{234}} \\
& +\frac{(\epsilon \epsilon)_{23}(\epsilon P)_{41} s_{34}}{s_{23} s_{234}}-\frac{(\epsilon \epsilon)_{23}(\epsilon P)_{45} s_{P_{1} 2}}{s_{23} s_{4 P_{5}}}+\frac{(\epsilon \epsilon)_{34}(\epsilon P)_{21}-(\epsilon \epsilon)_{24}(\epsilon P)_{31}+(\epsilon \epsilon)_{23}(\epsilon P)_{41}}{s_{234}} \\
& +\frac{(\epsilon \epsilon)_{34}(\epsilon k)_{23} s_{P_{1} 4}-(\epsilon \epsilon)_{24}(\epsilon k)_{32} s_{P_{1} 4}+(\epsilon \epsilon)_{23}(\epsilon k)_{42} s_{P_{1} 4}-(\epsilon \epsilon)_{23}(\epsilon P)_{41} s_{P_{1} 2}-(\epsilon \epsilon)_{23}(\epsilon P)_{45} s_{P_{1} 2}}{s_{23} s_{234}} \\
& +2 \times \frac{(\epsilon P)_{21}(\epsilon k)_{32}(\epsilon P)_{45}+(\epsilon P)_{25}(\epsilon P)_{31}(\epsilon P)_{45}+(\epsilon P)_{25}(\epsilon P)_{31}(\epsilon k)_{42}+(\epsilon P)_{21}(\epsilon P)_{31}(\epsilon P)_{45}-(1 \leftrightarrow 5)}{s_{34} s_{234}} \\
& +2 \times \frac{(\epsilon k)_{23}(\epsilon P)_{35}(\epsilon P)_{41}+(\epsilon k)_{32}(\epsilon P)_{45}(\epsilon P)_{21}-(1 \leftrightarrow 5)}{s_{23} s_{234}} .
\end{aligned}
$$

Specializing to four dimensions, this matches the spinor-helicity result provided in [34]. We note that this five-point amplitude $A_{5}\left(1_{\varphi}, 2_{g}, 3_{g}, 4_{g}, 5_{\varphi}\right)$ can also be computed using eq. (A.4) so that, alternatively,

$$
A_{5}\left(1_{\varphi}, 2_{g}, 3_{g}, 4_{g}, 5_{\varphi}\right)=\left.A_{5}\left(\mathbf{3}, 4, \boldsymbol{P}_{5}, P_{1}, 2\right)\right|_{\epsilon_{1}, \epsilon_{5}=(\overrightarrow{0}, 1)}
$$


It is now straightforward to move to any higher number of points, recursively. Using the result of the appendix we find the six-point amplitude

$$
\begin{aligned}
& A_{6}\left(1_{\varphi}, 2_{g}, 3_{g}, 4_{g}, 5_{g}, 6_{\varphi}\right)=\left.A_{6}\left(\boldsymbol{P}_{6}, P_{1}, \mathbf{2}, 3,4,5\right)\right|_{\epsilon_{1}, \epsilon_{6}=(\overrightarrow{0}, 1)} \\
& =\frac{A_{3}\left(\mathbf{2}, \boldsymbol{P}_{3: 6}^{\varphi}, P_{1}^{\varphi}\right) A_{5}\left(\boldsymbol{P}_{6}^{\varphi}, \boldsymbol{P}_{12}^{\varphi}, 3,4,5\right)}{s_{345 P_{6}}}+\sum_{M}\left[\frac{A_{3}\left(\boldsymbol{P}_{2: 5}^{\epsilon^{M}}, \boldsymbol{P}_{6}^{\varphi}, P_{1}^{\varphi}\right) A_{5}\left(\boldsymbol{P}_{61}^{\epsilon^{M}}, \mathbf{2}, 3,4,5\right)}{s_{2345}}\right. \\
& \left.+\frac{A_{3}\left(\boldsymbol{P}_{4: 1}^{\epsilon^{M}}, \mathbf{2}, 3\right) A_{5}\left(\boldsymbol{P}_{6}^{\varphi}, P_{1}^{\varphi}, \boldsymbol{P}_{23}^{\epsilon^{M}}, 4,5\right)}{s_{23}}-\frac{A_{4}\left(\boldsymbol{P}_{6}^{\varphi}, P_{1}^{\varphi}, \boldsymbol{P}_{2: 4}^{\epsilon^{M}}, 5\right) A_{4}\left(\boldsymbol{P}_{5: 1}^{\epsilon^{M}}, \mathbf{2}, 3,4\right)}{s_{234}}\right] .
\end{aligned}
$$

The longitudinal pieces have again cancelled, leaving a simple sum over intermediate polarizations and a very intuitive recursive structure, as shown. Although the explicit evaluation of this expression is straightforward, the resulting expression is lengthy and we do not reproduce it here.

\section{Kleiss-Kuijf decomposition}

While the method described in the previous section is straightforward and immediately generalizable to any number of gluons $n$, we wish to point out that an alternative track based on an expansion with analytically computed BCJ-numerators is of comparable simplicity. The trick is to compute the scattering of two massive scalar fields with massless gluons (eventually gravitons) by decomposing the reduced Pffafian in terms of a KleissKuijf (KK) basis [53] by using the Bern-Carrasco-Johansson (BCJ) numerators [54] for Yang-Mills theory. This useful technique was developed in $^{1}[50,55,56]$.

Let us recall that our first main goal is to calculate the amplitude

$$
A_{n}\left(1_{\varphi}, 2_{g}, 3_{g}, \ldots,(n-1)_{g}, n_{\varphi}\right)
$$

and in order to avoid dealing with the terms

$$
\frac{2 P_{1} \cdot P_{n}+2 \Delta_{1 n}}{\sigma_{1 n}} \text { and } \frac{2 P_{n} \cdot P_{1}+2 \Delta_{n 1}}{\sigma_{n 1}},
$$

we remove from the reduced Pfaffian the rows/columns $\{1, n\}$. Thus, we are looking for the following KK expansion

$$
\frac{(-1)^{1+n}}{\sigma_{1 n}} \times \operatorname{Pf}\left[(\Psi)_{1 n}^{1 n}\right]=\sum_{\rho \in S_{n-2}} N_{(1, \rho(2, \cdots, n-1), n)} \operatorname{PT}(1, \rho(2, \cdots, n-1), n),
$$

where $N_{(1, \rho(2, \cdots, n-1), n)}$ are the BCJ Yang-Mills numerators and $S_{n-2}$ is the group of the $(n-2)$ ! permutations of the set $\{2,3, \cdots, n-1\}$. As argued in [57], since the Pfaffian $\operatorname{Pf}\left[(\Psi)_{1 n}^{1 n}\right]$, is independent of the products $P_{1} \cdot P_{n}$ and $\Delta_{1 n}=\Delta_{n 1}=P_{1}^{2}$ the algorithm

\footnotetext{
${ }^{1}$ We thank Y. Geyer for sharing us her Mathematica package allowing us to carry out the master BCJ numerator evaluations in order to rewrite the reduced Pfaffian.
} 
proposed in ref. [56] can be applied. Therefore, the scattering between two massive scalar fields with $(n-2)$ gluons can be written as

$$
A_{n}\left(1_{\varphi}, 2_{g}, \ldots, n_{\varphi}\right)=\sum_{\rho \in S_{n-2}} m_{n}[12 \cdots n \mid 1 \rho(2 \cdots n-1) n] \times\left. N_{(1, \rho(2, \cdots, n-1), n)}\right|_{\epsilon_{1}, \epsilon_{n}=(\overrightarrow{0}, 1)},
$$

where the BCJ numerators $N_{(1, \rho(2, \cdots, n-1), n)}$ can be obtained by the algorithm developed in [56] and where $m_{n}[\alpha \mid \beta]$ is defined by

$$
m_{n}\left[\alpha_{1} \cdots \alpha_{n} \mid \beta_{1} \cdots \beta_{n}\right] \equiv \int d \mu_{n} \operatorname{PT}\left(\alpha_{1}, \ldots, \alpha_{n}\right) \times \operatorname{PT}\left(\beta_{1}, \ldots, \beta_{n}\right),
$$

with the massive measure $d \mu_{n}$ as given in (2.8).

To illustrate, let us consider the four-point amplitude $A_{4}\left(1_{\varphi}, 2_{g}, 3_{g}, 4_{\varphi}\right)$. From (3.3) we arrive at

$$
\begin{aligned}
A_{4}\left(1_{\varphi}, 2_{g}, 3_{g}, 4_{\varphi}\right)= & \left.N_{(1,2,3,4)}\right|_{\epsilon_{1}, \epsilon_{4}=(\overrightarrow{0}, 1)} \int d \mu_{4} \operatorname{PT}(1,2,3,4) \times \operatorname{PT}(1,2,3,4) \\
& +\left.N_{(1,3,2,4)}\right|_{\epsilon_{1}, \epsilon_{4}=(\overrightarrow{0}, 1)} \int d \mu_{4} \operatorname{PT}(1,2,3,4) \times \operatorname{PT}(1,3,2,4) .
\end{aligned}
$$

Now applying the method of ref. [56], the BCJ numerators are readily found to be given by

$$
\left.N_{(1,2,3,4)}\right|_{\epsilon_{1}, \epsilon_{4}=(\overrightarrow{0}, 1)}=-2(\epsilon P)_{21}(\epsilon P)_{34},\left.\quad N_{(1,3,2,4)}\right|_{\epsilon_{1}, \epsilon_{4}=(\overrightarrow{0}, 1)}=2(\epsilon P)_{21}(\epsilon P)_{31}+2(\epsilon F P)_{231}
$$

where we have fixed the reference ordering to be $(1,2,3,4)$. The massive integrals obtained in (3.5) are straightforward to do using the $\Lambda$-algorithm [39]. We find

$$
\begin{aligned}
& m_{4}[1234 \mid 1234]=\int d \mu_{4} \mathrm{PT}(1,2,3,4) \times \mathrm{PT}(1,2,3,4)=\frac{1}{s_{P_{1} 2}}+\frac{1}{s_{23}}, \\
& m_{4}[1234 \mid 1324]=\int d \mu_{4} \operatorname{PT}(1,2,3,4) \times \operatorname{PT}(1,3,2,4)=-\frac{1}{s_{23}} .
\end{aligned}
$$

For the four-point amplitude we therefore get

$$
A_{4}\left(1_{\varphi}, 2_{g}, 3_{g}, 4_{\varphi}\right)=\frac{-2(\epsilon P)_{21}(\epsilon P)_{34}}{s_{P_{1} 2}}+\frac{-2(\epsilon P)_{21}(\epsilon P)_{34}-\left(2(\epsilon P)_{21}(\epsilon P)_{31}+2(\epsilon F P)_{231}\right)}{s_{23}},
$$

which agrees with the result we found in equation (2.32). 


\section{Explicit BCJ numerators at five points}

This method easily generalizes. For two massive scalar legs and three gluons we need to evaluate

$$
A_{5}\left(1_{\varphi}, 2_{g}, 3_{g}, 4_{g}, 5_{\varphi}\right)=\sum_{\rho \in S_{3}} m_{5}[12345 \mid 1 \rho(234) 5] \times\left. N_{(1, \rho(2,3,4), 5)}\right|_{\epsilon_{1}, \epsilon_{5}=(\overrightarrow{0}, 1)} .
$$

The six BCJ-numerators $\left.N_{(1, \rho(2,3,4), 5)}\right|_{\epsilon_{1}, \epsilon_{5}=(\overrightarrow{0}, 1)}$ are given by

$$
\begin{aligned}
& \left.\left(\frac{1}{\sqrt{2}}\right) N_{(1,2,3,4,5)}\right|_{\epsilon_{1}, \epsilon_{5}=(\overrightarrow{0}, 1)}=-2(\epsilon P)_{21}(\epsilon P)_{45}\left[(\epsilon P)_{31}+(\epsilon k)_{32}\right], \\
& \left.\left(\frac{1}{\sqrt{2}}\right) N_{(1,4,2,3,5)}\right|_{\epsilon_{1}, \epsilon_{5}=(\overrightarrow{0}, 1)}=-2(\epsilon P)_{25}(\epsilon P)_{41}\left[(\epsilon P)_{21}+(\epsilon k)_{24}\right]-(\epsilon \epsilon)_{34}(\epsilon P)_{21} s_{P_{1} 4} \\
& +(\epsilon \epsilon)_{24}(\epsilon P)_{35} s_{P_{1} 4}, \\
& \left.\left(\frac{1}{\sqrt{2}}\right) N_{(1,3,4,2,5)}\right|_{\epsilon_{1}, \epsilon_{5}=(\overrightarrow{0}, 1)}=2(\epsilon P)_{25}(\epsilon P)_{31}\left[(\epsilon k)_{42}+(\epsilon P)_{45}\right]+(\epsilon \epsilon)_{23}\left[(\epsilon k)_{42}+(\epsilon P)_{45}\right] s_{P_{1} 3} \\
& -(\epsilon \epsilon)_{43}(\epsilon k)_{24} s_{P_{1} 3}-(\epsilon \epsilon)_{24}(\epsilon P)_{31} s_{P_{1} 34} \\
& -(\epsilon \epsilon)_{24}\left[(\epsilon k)_{32}+(\epsilon P)_{35}\right] s_{P_{1} 3} \text {, } \\
& \left.\left(\frac{1}{\sqrt{2}}\right) N_{(1,2,4,3,5)}\right|_{\epsilon_{1}, \epsilon_{5}=(\overrightarrow{0}, 1)}=-2(\epsilon P)_{21}(\epsilon P)_{35}\left[(\epsilon P)_{41}+(\epsilon k)_{42}\right]-(\epsilon \epsilon)_{34}(\epsilon P)_{21}\left(s_{P_{1} 4}+s_{24}\right) \text {, } \\
& \left.\left(\frac{1}{\sqrt{2}}\right) N_{(1,3,2,4,5)}\right|_{\epsilon_{1}, \epsilon_{5}=(\overrightarrow{0}, 1)}=-2(\epsilon P)_{31}(\epsilon P)_{45}\left[(\epsilon P)_{21}+(\epsilon k)_{23}\right]+(\epsilon \epsilon)_{23}(\epsilon P)_{45} s_{P_{1} 3}, \\
& \left.\left(\frac{1}{\sqrt{2}}\right) N_{(1,4,3,2,5)}\right|_{\epsilon_{1}, \epsilon_{5}=(\overrightarrow{0}, 1)}=2(\epsilon P)_{41}(\epsilon P)_{25}\left[(\epsilon k)_{32}+(\epsilon P)_{35}\right]-(\epsilon \epsilon)_{34}\left[(\epsilon P)_{21}+(\epsilon k)_{23}\right] s_{P_{1} 4} \\
& +(\epsilon \epsilon)_{24}\left[(\epsilon k)_{32}+(\epsilon P)_{35}\right] s_{P_{1} 4}-(\epsilon \epsilon)_{23}\left[(\epsilon k)_{42}+(\epsilon P)_{45}\right] s_{P_{1} 4} \\
& -(\epsilon \epsilon)_{23}(\epsilon P)_{41} s_{P_{1} 34} \text {, }
\end{aligned}
$$

where we have fixed the reference ordering to be $(1,2,3,4,5)$.

Using again the $\Lambda$-algorithm [39], it is straightforward to compute, with two massive legs,

$$
\begin{aligned}
& m_{5}[12345 \mid 12345]=\frac{1}{s_{234} s_{34}}+\frac{1}{s_{P_{1} 2} s_{34}}+\frac{1}{s_{23} s_{4 P_{5}}}+\frac{1}{s_{4 P_{5}} s_{P_{1} 2}}+\frac{1}{s_{23} s_{234}}, \\
& m_{5}[12345 \mid 14235]=-\frac{1}{s_{23} s_{234}}, \\
& m_{5}[12345 \mid 13425]=-\frac{1}{s_{234} s_{34}}, \\
& m_{5}[12345 \mid 12435]=-\frac{1}{s_{234} s_{34}}-\frac{1}{s_{P_{1} 2} s_{34}}, \\
& m_{5}[12345 \mid 13245]=-\frac{1}{s_{23} s_{4 P_{5}}}-\frac{1}{s_{23} s_{234}}, \\
& m_{5}[12345 \mid 14325]=\frac{1}{s_{234} s_{34}}+\frac{1}{s_{23} s_{234}} .
\end{aligned}
$$

After substituting eqs. (3.9) and (3.10) into (3.8) one can check that the result matches the one given in eq. (2.34).

This method does have the drawback for $n$ large that the number of BCJ numerators grow in a factorial way. For instance, to compute the six and seven-point amplitudes one needs to calculate $4 !=24$ and $5 !=120$ numerators, respectively. 


\section{Two massive scalars and gravitons}

In the previous sections, we have shown different methods for efficient evaluation of scattering amplitudes of two massive scalar fields and $(n-2)$ gluons. Staying within the CHY-framework as in section 2, one could similarly express the amplitude of the scattering among two massive scalars $(\varphi)$ and gravitons $\left(h_{a}\right)$ through $[50,58,59]$,

$$
\mathcal{M}_{n}(2 \varphi,(n-2) h)=\left.\int d \mu_{n} \operatorname{Pf}^{\prime} \Psi_{n}\right|_{\epsilon_{1}, \epsilon_{n}=(\overrightarrow{0}, 1)} \times\left.\operatorname{Pf}^{\prime} \Psi_{n}\right|_{\epsilon_{1}, \epsilon_{n}=(\overrightarrow{0}, 1)},
$$

where the gravitons are identified as, $h_{a}^{\mu \nu} \equiv \epsilon_{a}^{\mu} \epsilon_{a}^{\nu}$ and using the same massive measure defined in (2.8). Similarly, one can use a KK-decomposition analogous to what we explained above for the case of gluons in (3.3), and write

$$
\mathcal{M}_{n}(2 \varphi,(n-2) h)=\left.\sum_{\substack{\rho \in S_{n-2} \\ \delta \in S_{n-2}}} N_{(1, \rho, n)}\right|_{\epsilon_{1}, \epsilon_{n}=(\overrightarrow{0}, 1)} \times m_{n}[1 \rho n \mid 1 \delta n] \times\left. N_{(1, \delta, n)}\right|_{\epsilon_{1}, \epsilon_{n}=(\overrightarrow{0}, 1)} .
$$

However, by using the Kawai-Lewellen-Tye (KLT) [28] relations at the amplitude level, it seems much more straightforward to find the scattering between two massive scalar and $(n-2)$ gravitons by use of the momentum kernel [30-32], i.e,

$$
\begin{gathered}
\mathcal{M}_{n}(2 \varphi,(n-2) h)=(-1)^{n-3} \sum_{\substack{\alpha \in S_{n-3} \\
\beta \in S_{n-3}}} A_{n}\left(1_{\varphi}, \alpha_{g},(n-1)_{g}, n_{\varphi}\right) \times \mathcal{S}[\alpha \mid \beta]_{k_{1}} \\
\times A_{n}\left(n_{\varphi},(n-1)_{g}, \beta_{g}, 1_{\varphi}\right) .
\end{gathered}
$$

Here $A_{n}$ is an amplitude of two massive scalars and $(n-2)$ gluons as defined in (2.22), and the momentum kernel $\mathcal{S}[\alpha \mid \beta]$ is

$$
\mathcal{S}\left[i_{1}, \ldots, i_{k} \mid j_{1}, \ldots, j_{k}\right]_{k_{1}} \equiv \prod_{t=1}^{k}\left(s_{i_{t} 1}+\sum_{q>t}^{k} \Theta\left(i_{t}, i_{q}\right) s_{i_{t}, i_{q}}\right)
$$

where $\Theta$ is the step function. For instance, for the four-point amplitude we immediately get

$$
\mathcal{M}_{4}(2 \varphi, 2 h)=A_{4}\left(1_{\varphi}, 3_{g}, 2_{g}, 4_{\varphi}\right) \times \mathcal{S}[3 \mid 2] \times A_{4}\left(1_{\varphi}, 2_{g}, 3_{g}, 4_{\varphi}\right),
$$

where $\mathcal{S}[3 \mid 2]=-s_{23}$, thus using the result found in (2.32), one has

$$
\begin{aligned}
\mathcal{M}_{4}(2 \varphi, 2 h)= & {\left[\frac{2(\epsilon P)_{31}(\epsilon k)_{23} s_{P_{1} 3}-2(\epsilon F P)_{321} s_{P_{1} 3}-2(\epsilon P)_{31}(\epsilon P)_{24} s_{23}}{s_{P_{1} 3} s_{23}}\right] \times\left(-s_{23}\right) } \\
& \times\left[\frac{2(\epsilon P)_{21}(\epsilon k)_{32} s_{P_{1} 2}-2(\epsilon F P)_{231} s_{P_{1} 2}-2(\epsilon P)_{21}(\epsilon P)_{34} s_{23}}{s_{P_{1} 2} s_{23}}\right] \\
= & -\frac{\left[2(\epsilon P)_{24}(\epsilon P)_{31} s_{P_{1} 2}+2(\epsilon P)_{21}(\epsilon P)_{34} s_{2 P_{4}}+(\epsilon \epsilon)_{23} s_{P_{1} 2} s_{2 P_{4}}\right]^{2}}{s_{P_{1} 2} s_{23} s_{2 P_{4}}}
\end{aligned}
$$

which is the correct 4-point amplitude. Higher order amplitudes follow by KLT-squaring analogously. 


\section{Conclusion}

We have presented different methods to compute the tree-level scattering amplitudes of two massive scalars and an in principle arbitrary number of gravitons in $D$-dimensions. These are the tree-level amplitudes needed to obtain the classical two-body scattering of two massive objects without spin in general relativity through the use of unitarity. The most economical method appears to be the one based on a new set of recursive relations that can be derived from the so-called $\Lambda$-algorithm (or double cover) in the CHY-formalism. In this method one first defines an extension of scattering amplitudes where one external leg is taken off-shell (defining, effectively, a current in the case of Yang-Mills theory) and then glues off-shell legs together by an appropriate polarization sum. We have proven a particular simplification in comparison to the pure Yang-Mills case when the amplitude contains two massive scalar legs: a sum over longitudinal polarizations cancels exactly. The resulting amplitude relations for two massive scalars and any number of on-shell gluons thus becomes surprisingly simple.

Although a similar technique can be used to compute amplitudes of two massive scalars with an arbitrary number of gravitons we have found it economical to simply use KLTsquaring in order to obtain these. Again, they are then provided in $D$-dimensions and with arbitrary polarization tensors.

We have checked our general recursive formula up to six points with existing expressions in the literature for the case $D=4$, always finding complete agreement. An interesting observation is the possibility of establishing a new on-shell set of recursion relations for these amplitudes based on BCFW-recursion combined with the double-cover analysis of the $\Lambda$-algorithm. This will be discussed elsewhere.

\section{Acknowledgments}

We would like to thank Y. Geyer and J. Agerskov for helpful discussions. This project has received funding from the European Union's Horizon 2020 research and innovation programme under the Marie Sklodowska-Curie grant agreement No. 764850 "SAGEX" and has also been supported in part by the Danish National Research Foundation (DNRF91). H.G. acknowledges partial support from University Santiago de Cali (USC). 


\section{A Higher-point Yang-Mills amplitudes with massive legs}

Here we present details of the main ingredients that go into the computation of the massive five-point gluon amplitudes: $A_{5}\left(\boldsymbol{P}_{1}, P_{2}, \boldsymbol{P}_{3}, 4,5\right), A_{5}\left(\boldsymbol{P}_{1}, 2, \boldsymbol{P}_{3}, P_{4}, 5\right)$ and $A_{5}\left(\boldsymbol{P}_{1}, \boldsymbol{P}_{2}, P_{3}, 4,5\right)$.

Using the method developed in [43], the factorization decomposition of $A_{5}\left(\boldsymbol{P}_{1}, P_{2}, \boldsymbol{P}_{3}\right.$, $4,5)$ becomes

$$
\begin{aligned}
A_{5}\left(\boldsymbol{P}_{1}, P_{2}, \boldsymbol{P}_{3}, 4,5\right)=(-1) \times \sum_{M}\left\{\frac{A_{3}\left(\boldsymbol{P}_{5: 2}^{\epsilon^{M}}, \boldsymbol{P}_{3}, 4\right) \times A_{4}\left(\boldsymbol{P}_{1}, P_{2}, \boldsymbol{P}_{34}^{\epsilon^{M}}, 5\right)}{s_{P_{3} 4}}\right. \\
\left.+\frac{A_{3}\left(\boldsymbol{P}_{3: 5}^{\epsilon^{M}}, \boldsymbol{P}_{1}, P_{2}\right) \times A_{4}\left(\boldsymbol{P}_{12}^{\epsilon^{M}}, \boldsymbol{P}_{3}, 4,5\right)}{s_{P_{3} 45}}+\frac{A_{3}\left(\boldsymbol{P}_{3}, \boldsymbol{P}_{4: 1}^{\epsilon^{M}}, P_{2}\right) \times A_{4}\left(\boldsymbol{P}_{1}, \boldsymbol{P}_{23}^{\epsilon^{M}}, 4,5\right)}{s_{45 P_{1}}}\right\} \\
+2 \sum_{L}\left\{\frac{A_{3}\left(\boldsymbol{P}_{513}^{\epsilon^{L}}, \boldsymbol{P}_{2}, 4\right)}{s_{P_{2} 4}} \times A_{4}\left(\boldsymbol{P}_{1}, P_{3}, \boldsymbol{P}_{24}^{\epsilon^{L}}, 5\right)+\frac{A_{4}\left(\boldsymbol{P}_{13}^{\epsilon^{L}}, \boldsymbol{P}_{2}, 4,5\right)}{s_{P_{2} 45}}\right. \\
\left.\times A_{3}\left(\boldsymbol{P}_{245}^{\epsilon^{L}}, \boldsymbol{P}_{1}, P_{3}\right)\right\},
\end{aligned}
$$

where we have written $A_{5}\left(\boldsymbol{P}_{1}, P_{2}, \boldsymbol{P}_{3}, 4,5\right)$ in terms of the smaller amplitudes, $A_{3}\left(\boldsymbol{P}_{a}, \boldsymbol{P}_{b}\right.$, $\left.P_{c}\right), A_{4}\left(\boldsymbol{P}_{a}, P_{b}, \boldsymbol{P}_{c}, d\right)$ and $A_{4}\left(\boldsymbol{P}_{a}, \boldsymbol{P}_{b}, P_{c}, d\right)$. As in the four-point case, we must use the identities in (2.17) and (2.18).

It is straightforward to find the longitudinal contributions,

$$
\begin{aligned}
- & 2 \sum_{L}\left[\frac{A_{3}\left(\boldsymbol{P}_{513}^{\epsilon_{13}^{L}}, \boldsymbol{P}_{2}, 4\right)}{s_{P_{2}}} \times A_{4}\left(\boldsymbol{P}_{1}, P_{3}, \boldsymbol{P}_{24}^{\epsilon^{L}}, 5\right)\right] \\
= & -\sqrt{2}\left(\epsilon_{2} \cdot \epsilon_{4}\right) \times \frac{s_{5 P_{13}}\left(\epsilon_{1} \cdot F_{5} \cdot \epsilon_{3}\right)+2\left(\epsilon_{1} \cdot \epsilon_{3}\right)\left(P_{1} \cdot F_{5} \cdot P_{3}\right)}{s_{5 P_{13}} s_{P_{1} 5}},
\end{aligned}
$$

and

$$
\begin{aligned}
& -2 \sum_{L}\left[\frac{A_{4}\left(\boldsymbol{P}_{13}^{\epsilon^{L}}, \boldsymbol{P}_{2}, 4,5\right)}{s_{P_{2}} 45} \times A_{3}\left(\boldsymbol{P}_{245}^{\epsilon^{L}}, \boldsymbol{P}_{1}, P_{3}\right)\right] \\
& =-\sqrt{2}\left(\epsilon_{1} \cdot \epsilon_{3}\right) \times \frac{s_{5 P_{24}}\left(\epsilon_{2} \cdot F_{5} \cdot \epsilon_{4}\right)+2\left(\epsilon_{2} \cdot \epsilon_{4}\right)\left(P_{2} \cdot F_{5} \cdot k_{4}\right)}{s_{5 P_{24}} s_{45}} .
\end{aligned}
$$

Similarly, the amplitude $A_{5}\left(\boldsymbol{P}_{1}, 2, \boldsymbol{P}_{3}, P_{4}, 5\right)$ is factorized according to

$$
\begin{gathered}
A_{5}\left(\boldsymbol{P}_{1}, 2, \boldsymbol{P}_{3}, P_{4}, 5\right)=(-1) \times \sum_{M}\left\{\frac{A_{3}\left(\boldsymbol{P}_{5: 2}^{\epsilon^{M}}, \boldsymbol{P}_{3}, P_{4}\right) \times A_{4}\left(\boldsymbol{P}_{1}, 2, \boldsymbol{P}_{34}^{\epsilon^{M}}, 5\right)}{s_{P_{3} P_{4}}+2 \Delta_{34}}+\right. \\
\left.\frac{A_{3}\left(\boldsymbol{P}_{3: 5}^{\epsilon^{M}}, \boldsymbol{P}_{1}, 2\right) \times A_{4}\left(\boldsymbol{P}_{12}^{\epsilon^{M}}, \boldsymbol{P}_{3}, P_{4}, 5\right)}{s_{P_{3} P_{4} 5}+2 \Delta_{34}}+\frac{A_{3}\left(\boldsymbol{P}_{3}, \boldsymbol{P}_{4: 1}^{\epsilon^{M}}, 2\right) \times A_{4}\left(\boldsymbol{P}_{1}, \boldsymbol{P}_{23}^{\epsilon^{M}}, P_{4}, 5\right)}{s_{P_{4}} 5 P_{1}+2 \Delta_{14}}\right\} \\
+2 \sum_{L}\left\{\frac{A_{3}\left(\boldsymbol{P}_{513}^{\epsilon_{1}^{L}}, \mathbf{2}, P_{4}\right)}{s_{2 P_{4}}+2 \Delta_{14}+2 \Delta_{34}} A_{4}\left(\boldsymbol{P}_{1}, P_{3}, \boldsymbol{P}_{24}^{\epsilon^{L}}, 5\right)+\frac{A_{4}\left(\boldsymbol{P}_{13}^{\epsilon^{L}}, \mathbf{2}, P_{4}, 5\right)}{s_{2 P_{4} 5}+2 \Delta_{14}+2 \Delta_{34}}\right. \\
\left.\times A_{3}\left(\boldsymbol{P}_{245}^{\epsilon^{L}}, \boldsymbol{P}_{1}, P_{3}\right)\right\}
\end{gathered}
$$


where we have used (2.6), namely

$$
\Delta_{13}=\frac{P_{1}^{2}+P_{3}^{2}-P_{4}^{2}}{2}, \quad \Delta_{14}=\frac{P_{1}^{2}-P_{3}^{2}+P_{4}^{2}}{2}, \quad \Delta_{34}=\frac{-P_{1}^{2}+P_{3}^{2}+P_{4}^{2}}{2} .
$$

We also recall that $\Delta_{14}+\Delta_{34}=P_{4}^{2}$. It is now straightforward to verify that the longitudinal contributions in (A.4) are identical to the one evaluated above, i.e.,

$$
-2 \sum_{L}\left[\frac{A_{3}\left(\boldsymbol{P}_{513}^{\epsilon^{L}}, \mathbf{2}, P_{4}\right)}{s_{P_{4} 2}+2 \Delta_{14}+2 \Delta_{34}} \times A_{4}\left(\boldsymbol{P}_{1}, P_{3}, \boldsymbol{P}_{24}^{\epsilon^{L}}, 5\right)\right]=(\text { A. } 2)
$$

and

$$
-2 \sum_{L}\left[\frac{A_{4}\left(\boldsymbol{P}_{13}^{\epsilon^{L}}, \mathbf{2}, P_{4}, 5\right)}{s_{P_{4} 25}+2 \Delta_{14}+2 \Delta_{34}} \times A_{3}\left(\boldsymbol{P}_{245}^{\epsilon^{L}}, \boldsymbol{P}_{1}, P_{3}\right)\right]=(\mathrm{A} .3) .
$$

Finally, we are able to expand the amplitude, $A_{5}\left(\boldsymbol{P}_{1}, \boldsymbol{P}_{2}, P_{3}, P_{4}, 5\right)$, in terms of the two previous ones, $A_{5}\left(\boldsymbol{P}_{a}, P_{b}, \boldsymbol{P}_{c}, d, e\right)$ and $A_{5}\left(\boldsymbol{P}_{a}, b, \boldsymbol{P}_{c}, P_{d}, e\right)$. Using the BCJ-like identity [44, 45],

$s_{P_{3} 45} \mathrm{PT}(3,4,5,1,2)+\left(s_{P_{3} 45}+s_{P_{1} 5}\right) \mathrm{PT}(3,4,1,5,2)+\left(s_{P_{3} 45}+s_{P_{1} P_{45}}\right) \mathrm{PT}(3,1,4,5,2)=0$, the amplitude, $A_{5}\left(\boldsymbol{P}_{1}, \boldsymbol{P}_{2}, P_{3}, 4,5\right)$, turns into

$$
\begin{aligned}
& A_{5}\left(\boldsymbol{P}_{1}, \boldsymbol{P}_{2}, P_{3}, 4,5\right) \\
& \quad=-\left(1+\frac{s_{P_{1} P_{45}}}{s_{P_{3} 45}}\right) A_{5}\left(\boldsymbol{P}_{2}, P_{3}, \boldsymbol{P}_{1}, 4,5\right)-\left(1+\frac{s_{P_{12} 6}}{s_{P_{3} 45}}\right) A_{5}\left(\boldsymbol{P}_{1}, 5, \boldsymbol{P}_{2}, P_{3}, 4\right) .
\end{aligned}
$$

Finally, let us show how to compute the six-point amplitude, $A_{6}\left(\boldsymbol{P}_{1}, P_{2}, \boldsymbol{P}_{3}, 4,5,6\right)$. The factorization decomposition of $A_{6}\left(\boldsymbol{P}_{1}, P_{2}, \boldsymbol{P}_{3}, 4,5,6\right)$ is given by

$$
\begin{aligned}
& A_{6}\left(\boldsymbol{P}_{1}, P_{2}, \boldsymbol{P}_{3}, 4,5,6\right)=\sum_{M}\left\{\frac{A_{3}\left(\boldsymbol{P}_{3}, \boldsymbol{P}_{4: 1}^{\epsilon^{M}}, P_{2}\right) \times A_{5}\left(\boldsymbol{P}_{1}, \boldsymbol{P}_{23}^{\epsilon^{M}}, 4,5,6\right)}{s_{456 P_{1}}}\right. \\
& +\frac{A_{3}\left(\boldsymbol{P}_{3: 6}^{\epsilon^{M}}, \boldsymbol{P}_{1}, P_{2}\right) \times A_{5}\left(\boldsymbol{P}_{12}^{\epsilon^{M}}, \boldsymbol{P}_{3}, 4,5,6\right)}{s_{P_{3} 456}}+\frac{A_{3}\left(\boldsymbol{P}_{5: 2}^{\epsilon^{M}}, \boldsymbol{P}_{3}, 4\right) \times A_{5}\left(\boldsymbol{P}_{1}, P_{2}, \boldsymbol{P}_{34}^{\epsilon^{M}}, 5,6\right)}{s_{P_{3}} 4} \\
& \left.-\frac{A_{4}\left(\boldsymbol{P}_{1}, P_{2}, \boldsymbol{P}_{3: 5}^{\epsilon^{M}}, 6\right) \times A_{4}\left(\boldsymbol{P}_{6: 2}^{\epsilon^{M}}, \boldsymbol{P}_{3}, 4,5\right)}{s_{P_{3} 45}}\right\} \\
& -2 \sum_{L}\left\{\frac{A_{3}\left(\boldsymbol{P}_{3: 6}^{\epsilon^{L}}, \boldsymbol{P}_{1}, P_{2}\right) \times A_{5}\left(\boldsymbol{P}_{12}^{\epsilon^{L}}, \boldsymbol{P}_{3}, 4,5,6\right)}{s_{P_{3} 456}}+\frac{A_{3}\left(\boldsymbol{P}_{5: 2}^{\epsilon^{L}}, \boldsymbol{P}_{3}, 4\right) \times A_{5}\left(\boldsymbol{P}_{1}, P_{2}, \boldsymbol{P}_{34}^{\epsilon^{L}}, 5,6\right)}{s_{P_{3}} 4}\right. \\
& \left.-\frac{A_{4}\left(\boldsymbol{P}_{1}, P_{2}, \boldsymbol{P}_{3: 5}^{\epsilon^{L}}, 6\right) \times A_{4}\left(\boldsymbol{P}_{6: 2}^{\epsilon^{L}}, \boldsymbol{P}_{3}, 4,5\right)}{s_{P_{3} 45}}\right\}\left.\right|_{\substack{2 \leftrightarrow 3 \\
\left(\epsilon_{\alpha} \cdot P_{A}\right)=-\left(\epsilon_{\alpha} \cdot P_{\bar{A}}\right) \\
\left(\epsilon_{\alpha} \cdot P_{\alpha}\right)=0}},
\end{aligned}
$$

where $2 \leftrightarrow 3$ means the changing of the two labels, $\alpha=1,3$ and $P_{\bar{A}}$ is the complement of $P_{A}$ (by the momentum conservation condition, $P_{A}+P_{\bar{A}}=0$ ). For example, $P_{A}$ is given by $P_{2456}, P_{24}$ and $P_{245}$ in the last three term in (A.7), respectively, therefore, $P_{\bar{A}}$ is $P_{13}, P_{1356}$ and $P_{136}$. Additionally, the identities in (2.17) and (2.18) must be used in the above factorization expansion. 


\section{B Longitudinal contributions}

As we have observed in all special cases worked out in this paper, the longitudinal contributions to the factorized amplitudes with massive scalars always vanish identically. In this section we prove this important fact in all generality.

Let us consider a Yang-Mills $n$-point amplitude with up to three massive legs $A_{n}\left(\boldsymbol{P}_{n}, P_{1}, \boldsymbol{P}_{2}, 3, \ldots, n-1\right)$. Applying the factorization method, a generic longitudinal contribution is given by

$$
\sum_{L} \frac{A_{(n-i)+2}\left(\boldsymbol{P}_{n}, P_{2}, \boldsymbol{P}_{134 \ldots i}^{\epsilon^{L}}, i+1, \ldots, n-1\right) \times A_{i}\left(\boldsymbol{P}_{i+1 \ldots n 2}^{\epsilon^{L}}, \boldsymbol{P}_{1}, 3,4, \ldots, i\right)}{s_{P_{1} 34 \ldots i}},
$$

where the two amplitudes are sewn together by the rule

$$
\sum_{L} \epsilon_{i}^{L \mu} \epsilon_{j}^{L \nu}=\frac{P_{i}^{\mu} P_{j}^{\nu}}{P_{i} \cdot P_{j}+P_{n}^{2}-P_{2}^{2}} .
$$

We can now show the following:

Under the condition $\epsilon_{1}=\epsilon_{n}=(\overrightarrow{0}, 1)$, the amplitudes, $A_{(n-i)+2}\left(\boldsymbol{P}_{n}, P_{2}, \boldsymbol{P}_{134 \ldots i}^{\epsilon^{L}}, i+\right.$ $1, \ldots, n-1)$ and $A_{i}\left(\boldsymbol{P}_{i+1 \ldots n 2}^{\epsilon^{L}}, \boldsymbol{P}_{1}, 3,4, \ldots, i\right)$ vanish identically.

The proof of this proposition is straightforward.

Let us consider the amplitude, $A_{i}\left(\boldsymbol{P}_{i+1 \ldots n 2}^{\epsilon^{L}}, \boldsymbol{P}_{1}, 3,4, \ldots, i\right)$. From the notation introduced in the main text, it is clear that the reduced matrix $\left[\left(\Psi_{i}\right)_{P_{i+1 \ldots n 2} P_{1}}^{P_{i+1 \ldots n 2} P_{1}}\right]$, has a row (column) given by the vector

$$
\left.\left(\frac{\epsilon_{1} \cdot k_{2}}{\sigma_{12}}, \cdots, \frac{\epsilon_{1} \cdot k_{i}}{\sigma_{1 i}}, \frac{\epsilon_{1} \cdot \epsilon_{i+1 \ldots n 2}^{L}}{\sigma_{1 P_{i+1 \ldots n 2}}}, 0, \frac{\epsilon_{1} \cdot \epsilon_{2}}{\sigma_{12}}, \cdots, \frac{\epsilon_{1} \cdot \epsilon_{i}}{\sigma_{1 i}}\right)\right|_{\epsilon_{1}, \epsilon_{n}=(\overrightarrow{0}, 1)}=\left(0, \cdots, 0, \frac{\epsilon_{1} \cdot \epsilon_{i+1 \ldots n 2}^{L}}{\sigma_{1 P_{i+1 \ldots n 2}}}, 0, \cdots, 0\right) .
$$

Since $\epsilon_{i+1 \ldots n 2}^{L}$ is proportional to $P_{i+1 \ldots n 2}=k_{i+1}+\cdots+k_{n}+P_{2}$ it follows that

$$
\frac{\epsilon_{1} \cdot \epsilon_{i+1 \ldots n 2}^{L}}{\sigma_{1 P_{i+1 \ldots n 2}}} \propto \frac{\epsilon_{1} \cdot P_{i+1 \ldots n 2}}{\sigma_{1 P_{i+1 \ldots n 2}}}=0
$$

using that $\left(\epsilon_{1} \cdot k_{i}\right)=0$. Therefore, $A_{i}\left(\boldsymbol{P}_{i+1 \ldots n 2}^{\epsilon^{L}}, \boldsymbol{P}_{1}, 3,4, \ldots, i\right)$ vanishes trivially for, $\epsilon_{1}=$ $\epsilon_{n}=(\overrightarrow{0}, 1)$. The essential property that makes these contributions vanish is the fact that the polarization vectors associated with what become massive scalars live in a higher dimensional space with no overlap with the momenta of the $D$-dimensional space.

The same argument works for $A_{(n-i)+2}\left(\boldsymbol{P}_{n}, P_{2}, \boldsymbol{P}_{134 \ldots i}^{\epsilon^{L}}, i+1, \ldots, n-1\right)$.

Open Access. This article is distributed under the terms of the Creative Commons Attribution License (CC-BY 4.0), which permits any use, distribution and reproduction in any medium, provided the original author(s) and source are credited. 


\section{References}

[1] D. Neill and I.Z. Rothstein, Classical Space-Times from the S Matrix, Nucl. Phys. B 877 (2013) 177 [arXiv:1304.7263] [INSPIRE].

[2] B.R. Holstein and A. Ross, Spin Effects in Long Range Gravitational Scattering, arXiv:0802.0716 [INSPIRE].

[3] N.E.J. Bjerrum-Bohr, J.F. Donoghue and P. Vanhove, On-shell Techniques and Universal Results in Quantum Gravity, JHEP 02 (2014) 111 [arXiv: 1309.0804] [INSPIRE].

[4] V. Vaidya, Gravitational spin Hamiltonians from the S matrix, Phys. Rev. D 91 (2015) 024017 [arXiv: 1410.5348] [INSPIRE].

[5] N.E.J. Bjerrum-Bohr, J.F. Donoghue, B.R. Holstein, L. Planté and P. Vanhove, Bending of Light in Quantum Gravity, Phys. Rev. Lett. 114 (2015) 061301 [arXiv:1410.7590] [INSPIRE].

[6] N.E.J. Bjerrum-Bohr, J.F. Donoghue, B.R. Holstein, L. Plante and P. Vanhove, Light-like Scattering in Quantum Gravity, JHEP 11 (2016) 117 [arXiv:1609.07477] [INSPIRE].

[7] R.A. Porto, The effective field theorist's approach to gravitational dynamics, Phys. Rept. 633 (2016) 1 [arXiv: 1601.04914] [INSPIRE].

[8] T. Damour, Gravitational scattering, post-Minkowskian approximation and Effective One-Body theory, Phys. Rev. D 94 (2016) 104015 [arXiv:1609.00354] [INSPIRE].

[9] F. Cachazo and A. Guevara, Leading Singularities and Classical Gravitational Scattering, arXiv:1705.10262 [INSPIRE].

[10] A. Guevara, Holomorphic Classical Limit for Spin Effects in Gravitational and Electromagnetic Scattering, JHEP 04 (2019) 033 [arXiv: 1706.02314] [INSPIRE].

[11] T. Damour, High-energy gravitational scattering and the general relativistic two-body problem, Phys. Rev. D 97 (2018) 044038 [arXiv:1710.10599] [InSPIRE].

[12] N.E.J. Bjerrum-Bohr, P.H. Damgaard, G. Festuccia, L. Planté and P. Vanhove, General Relativity from Scattering Amplitudes, Phys. Rev. Lett. 121 (2018) 171601 [arXiv: 1806.04920] [INSPIRE].

[13] M. Levi, Effective Field Theories of Post-Newtonian Gravity: A comprehensive review, arXiv: 1807.01699 [INSPIRE].

[14] C. Cheung, I.Z. Rothstein and M.P. Solon, From Scattering Amplitudes to Classical Potentials in the Post-Minkowskian Expansion, Phys. Rev. Lett. 121 (2018) 251101 [arXiv: 1808.02489] [INSPIRE].

[15] M.-Z. Chung, Y.-T. Huang, J.-W. Kim and S. Lee, The simplest massive S-matrix: from minimal coupling to Black Holes, JHEP 04 (2019) 156 [arXiv:1812.08752] [INSPIRE].

[16] Z. Bern, C. Cheung, R. Roiban, C.-H. Shen, M.P. Solon and M. Zeng, Scattering Amplitudes and the Conservative Hamiltonian for Binary Systems at Third Post-Minkowskian Order, Phys. Rev. Lett. 122 (2019) 201603 [arXiv:1901.04424] [INSPIRE].

[17] Z. Bern, C. Cheung, R. Roiban, C.-H. Shen, M.P. Solon and M. Zeng, Black Hole Binary Dynamics from the Double Copy and Effective Theory, JHEP 10 (2019) 206 [arXiv: 1908.01493] [INSPIRE].

[18] A. Antonelli, A. Buonanno, J. Steinhoff, M. van de Meent and J. Vines, Energetics of two-body Hamiltonians in post-Minkowskian gravity, Phys. Rev. D 99 (2019) 104004 [arXiv: 1901.07102] [INSPIRE]. 
[19] A. Cristofoli, N.E.J. Bjerrum-Bohr, P.H. Damgaard and P. Vanhove, Post-Minkowskian Hamiltonians in general relativity, Phys. Rev. D 100 (2019) 084040 [arXiv:1906.01579] [INSPIRE].

[20] A. Koemans Collado, P. Di Vecchia and R. Russo, Revisiting the second post-Minkowskian eikonal and the dynamics of binary black holes, Phys. Rev. D 100 (2019) 066028 [arXiv: 1904.02667] [INSPIRE].

[21] B. Maybee, D. O'Connell and J. Vines, Observables and amplitudes for spinning particles and black holes, arXiv:1906.09260 [INSPIRE].

[22] Y. Iwasaki, Quantum theory of gravitation vs. classical theory: fourth-order potential, Prog. Theor. Phys. 46 (1971) 1587 [INSPIRE].

[23] B.R. Holstein and J.F. Donoghue, Classical physics and quantum loops, Phys. Rev. Lett. 93 (2004) 201602 [hep-th/0405239] [INSPIRE].

[24] D.A. Kosower, B. Maybee and D. O'Connell, Amplitudes, Observables and Classical Scattering, JHEP 02 (2019) 137 [arXiv:1811.10950] [INSPIRE].

[25] J.F. Donoghue, General relativity as an effective field theory: The leading quantum corrections, Phys. Rev. D 50 (1994) 3874 [gr-qc/9405057] [INSPIRE].

[26] N.E.J. Bjerrum-Bohr, J.F. Donoghue and B.R. Holstein, Quantum gravitational corrections to the nonrelativistic scattering potential of two masses, Phys. Rev. D 67 (2003) 084033 [Erratum ibid. D 71 (2005) 069903] [hep-th/0211072] [INSPIRE].

[27] N.E.J. Bjerrum-Bohr, J.F. Donoghue and B.R. Holstein, Quantum corrections to the Schwarzschild and Kerr metrics, Phys. Rev. D 68 (2003) 084005 [Erratum ibid. D 71 (2005) 069904] [hep-th/0211071] [INSPIRE].

[28] H. Kawai, D.C. Lewellen and S.H.H. Tye, A Relation Between Tree Amplitudes of Closed and Open Strings, Nucl. Phys. B 269 (1986) 1 [InSPIRE].

[29] Z. Bern, L.J. Dixon, M. Perelstein and J.S. Rozowsky, Multileg one loop gravity amplitudes from gauge theory, Nucl. Phys. B 546 (1999) 423 [hep-th/9811140] [INSPIRE].

[30] N.E.J. Bjerrum-Bohr, P.H. Damgaard, B. Feng and T. Sondergaard, Proof of Gravity and Yang-Mills Amplitude Relations, JHEP 09 (2010) 067 [arXiv:1007.3111] [INSPIRE].

[31] N.E.J. Bjerrum-Bohr, P.H. Damgaard, B. Feng and T. Sondergaard, Gravity and Yang-Mills Amplitude Relations, Phys. Rev. D 82 (2010) 107702 [arXiv: 1005.4367] [InSPIRE].

[32] N.E.J. Bjerrum-Bohr, P.H. Damgaard, T. Sondergaard and P. Vanhove, The Momentum Kernel of Gauge and Gravity Theories, JHEP 01 (2011) 001 [arXiv:1010.3933] [InSPIRE].

[33] S.D. Badger, E.W.N. Glover, V.V. Khoze and P. Svrček, Recursion relations for gauge theory amplitudes with massive particles, JHEP 07 (2005) 025 [hep-th/0504159] [INSPIRE].

[34] D. Forde and D.A. Kosower, All-multiplicity amplitudes with massive scalars, Phys. Rev. D 73 (2006) 065007 [hep-th/0507292] [INSPIRE].

[35] S.G. Naculich, Scattering equations and BCJ relations for gauge and gravitational amplitudes with massive scalar particles, JHEP 09 (2014) 029 [arXiv:1407.7836] [INSPIRE].

[36] S.G. Naculich, CHY representations for gauge theory and gravity amplitudes with up to three massive particles, JHEP 05 (2015) 050 [arXiv:1501.03500] [INSPIRE].

[37] F. Cachazo, S. He and E.Y. Yuan, Scattering of Massless Particles in Arbitrary Dimensions, Phys. Rev. Lett. 113 (2014) 171601 [arXiv: 1307.2199] [INSPIRE].

[38] F. Cachazo, S. He and E.Y. Yuan, Scattering Equations and Matrices: From Einstein To Yang-Mills, DBI and NLSM, JHEP 07 (2015) 149 [arXiv:1412.3479] [INSPIRE]. 
[39] H. Gomez, $\Lambda$ scattering equations, JHEP 06 (2016) 101 [arXiv: 1604.05373] [InSPIRE].

[40] C. Cardona and H. Gomez, Elliptic scattering equations, JHEP 06 (2016) 094 [arXiv: 1605.01446] [INSPIRE].

[41] N.E.J. Bjerrum-Bohr, P.H. Damgaard and H. Gomez, New Factorization Relations for Yang-Mills Amplitudes, Phys. Rev. D 99 (2019) 025014 [arXiv:1810.05023] [INSPIRE].

[42] N.E.J. Bjerrum-Bohr, H. Gomez and A. Helset, New factorization relations for nonlinear б-model amplitudes, Phys. Rev. D 99 (2019) 045009 [arXiv:1811.06024] [INSPIRE].

[43] H. Gomez, Scattering equations and a new factorization for amplitudes. Part I. Gauge theories, JHEP 05 (2019) 128 [arXiv:1810.05407] [INSPIRE].

[44] N.E.J. Bjerrum-Bohr, J.L. Bourjaily, P.H. Damgaard and B. Feng, Analytic representations of Yang-Mills amplitudes, Nucl. Phys. B 913 (2016) 964 [arXiv:1605.06501] [InSPIRE].

[45] C. Cardona, B. Feng, H. Gomez and R. Huang, Cross-ratio Identities and Higher-order Poles of CHY-integrand, JHEP 09 (2016) 133 [arXiv:1606.00670] [INSPIRE].

[46] N.E.J. Bjerrum-Bohr, J.L. Bourjaily, P.H. Damgaard and B. Feng, Manifesting Color-Kinematics Duality in the Scattering Equation Formalism, JHEP 09 (2016) 094 [arXiv: 1608.00006] [INSPIRE].

[47] T. Adamo, E. Casali and D. Skinner, Ambitwistor strings and the scattering equations at one loop, JHEP 04 (2014) 104 [arXiv:1312.3828] [INSPIRE].

[48] E. Casali and P. Tourkine, Infrared behaviour of the one-loop scattering equations and supergravity integrands, JHEP 04 (2015) 013 [arXiv: 1412.3787] [INSPIRE].

[49] Y. Geyer, L. Mason, R. Monteiro and P. Tourkine, One-loop amplitudes on the Riemann sphere, JHEP 03 (2016) 114 [arXiv:1511.06315] [InSPIRE].

[50] F. Cachazo, S. He and E.Y. Yuan, Scattering of Massless Particles: Scalars, Gluons and Gravitons, JHEP 07 (2014) 033 [arXiv: 1309.0885] [INSPIRE].

[51] L. Dolan and P. Goddard, Proof of the Formula of Cachazo, He and Yuan for Yang-Mills Tree Amplitudes in Arbitrary Dimension, JHEP 05 (2014) 010 [arXiv:1311.5200] [INSPIRE].

[52] H. Gomez and A. Helset, Scattering equations and a new factorization for amplitudes. Part II. Effective field theories, JHEP 05 (2019) 129 [arXiv: 1902.02633] [INSPIRE].

[53] R. Kleiss and H. Kuijf, Multi-Gluon Cross-sections and Five Jet Production at Hadron Colliders, Nucl. Phys. B 312 (1989) 616 [inSPIRE].

[54] Z. Bern, J.J.M. Carrasco and H. Johansson, New Relations for Gauge-Theory Amplitudes, Phys. Rev. D 78 (2008) 085011 [arXiv: 0805. 3993] [INSPIRE].

[55] C.S. Lam and Y.-P. Yao, Evaluation of the Cachazo-He-Yuan gauge amplitude, Phys. Rev. D 93 (2016) 105008 [arXiv: 1602.06419] [INSPIRE].

[56] C.-H. Fu, Y.-J. Du, R. Huang and B. Feng, Expansion of Einstein-Yang-Mills Amplitude, JHEP 09 (2017) 021 [arXiv: 1702.08158] [INSPIRE].

[57] Y. Geyer and R. Monteiro, Gluons and gravitons at one loop from ambitwistor strings, JHEP 03 (2018) 068 [arXiv:1711.09923] [INSPIRE].

[58] F. Cachazo, S. He and E.Y. Yuan, Scattering equations and Kawai-Lewellen-Tye orthogonality, Phys. Rev. D 90 (2014) 065001 [arXiv:1306.6575] [INSPIRE].

[59] F. Cachazo and H. Gomez, Computation of Contour Integrals on $\mathcal{M}_{0, n}$, JHEP 04 (2016) 108 [arXiv: 1505.03571] [INSPIRE].

[60] J. Plefka and W. Wormsbecher, New relations for graviton-matter amplitudes, Phys. Rev. D 98 (2018) 026011 [arXiv: 1804.09651] [INSPIRE]. 